\title{
SALUD INTERCULTURAL: EL CICLO VITAL EN LOS ANDES
}

\author{
Luis Alberto Santa María ${ }^{1,2, a}$
}

\begin{abstract}
RESUMEN
Se presenta las teorías e ideas más relevantes sobre el ciclo vital en los Andes, y su relación con el desarrollo humano y la salud. El ciclo vital no es un fenómeno inmutable, el número de etapas y su significado está pautado histórica y culturalmente. Se realiza un análisis comparativo con la teoría de Freud y Erikson. Se concluye que el aporte de la cultura andina se centra en: 1) Cada etapa del desarrollo humano está asociada a procesos productivos y reproductivos de la vida en relación, así como a un adecuado equilibrio entre derechos y deberes en la vida en sociedad; 2) La pareja es la unidad social básica, y no el individuo. Es un aporte para el diseño de intervenciones sanitarias con enfoque intercultural.
\end{abstract}

Palabras clave: Salud de poblaciones indígenas, Estadios del ciclo de vida (fuente: DeCS BIREME).

\section{INTERCULTURAL HEALTH: THE LIFE CYCLE STAGES IN THE ANDES}

\begin{abstract}
The most relevant theories and ideas about the life cycle in the Andes and their relationship with human development and health are presented. The life cycle is not an immutable phenomenon, the number of stages and its meaning is historically and culturally based. A comparative analysis with the theory of Freud and Erikson was made. It is concluded that the contribution of Andean culture focuses on: 1) Each stage of human development is associated with productive and reproductive processes of life in relation, as well as an adequate balance between rights and duties in life in society; 2) The couple is the basic social unit, and not the individual. It is a contribution to the design of health interventions with an intercultural approach.
\end{abstract}

Key words: Health of indigenous peoples, Life cycle stages (source: MeSH NLM).

\section{INTRODUCCIÓN}

La Organización Mundial de la Salud considera que: La identidad étnica es un determinante social que incide en diferentes áreas relacionadas con la prevención, promoción y atención de la salud (1), y recomienda: Incorporar la cultura del usuario en el proceso de atención de salud ${ }^{(1)}$.

En el "mundo occidental" se han desarrollado modelos que incorporan las ideas de los padres y la sociedad sobre el buen crecimiento del niño en la puericultura y sistemas de cuidado del niño (2). En el "mundo andino y amazónico", la construcción de estos modelos es una tarea pendiente para diseñar intervenciones sanitarias con enfoque intercultural. Cuando se habla de ciclo vital, se hace referencia al ciclo que comprende toda la vida humana, desde la concepción hasta la muerte, en el que se identifican tres crisis vitales: nacimiento, reproducción y muerte ${ }^{(3,4,5)}$.
En 1608, Guamán Poma de Ayala describió el ciclo vital conceptualizado durante el tiempo de los incas (Tabla 1). Cada etapa del ciclo vital estaba relacionada con una actividad productiva. Antes de los 5 años de edad, los niños ayudaban a sus padres en lo que podían. A partir de los 5 años de edad se les daba tareas de mayor complejidad (6). A esa edad, para los incas, un niño podía ser tributario, mientras para los españoles un niño menor de 5 años no podía ser tributario ${ }^{(7)}$.

En los censos realizados por los españoles entre la población inca, se encuentran palabras como huahua, paya, sipas, huayna, warma, con las que en la actualidad se determinan diferentes etapas del ciclo vital ${ }^{(7)}$. La edad no es el criterio seguido para determinar la pertenencia a cada etapa, sino la capacidad de cumplir con las funciones sociales establecidas.

\footnotetext{
Instituto Nacional de Salud. Lima, Perú.

Universidad Nacional Mayor de San Marcos. Lima, Perú.
}

Médico cirujano, doctor en Salud Publica.

El artículo es parte de la tesis de Santa María Juárez Luis Alberto. Sistema Cultural de Crianza en Ninamarca, Cusco. Tesis para optar el Grado de Doctor en Ciencias Sociales, especialidad en Antropologia. UNMSM. 2017.

Recibido: 06/03/2017 Aprobado: 07/06/2017 En línea: 28/06/2017 
Tabla 1. Ciclo vital descrito por Guaman Poma, 1608

\begin{tabular}{|c|c|c|}
\hline Edad aproximada & Varones & Mujeres \\
\hline De 1 día a 1 año & Guagua quiraupicoc: Bebitos de teta & $\begin{array}{l}\text { Llullu guagua huarmi. Igual que los bebitos de } \\
\text { su edad }\end{array}$ \\
\hline De 1 a 5 años & $\begin{array}{l}\text { Llullollac huanracuna. Fuera de la producción. } \\
\text { Niños dados al juego y para que otros los atiendan } \\
\text { y cuiden. }\end{array}$ & $\begin{array}{l}\text { Llucac huarmiguagua. Igual que los niños de su } \\
\text { edad. }\end{array}$ \\
\hline De 5 a 9 años & $\begin{array}{l}\text { Pucllacoc huaracuna. Ayudantes de sus padres, por } \\
\text { lo común cuidando a sus hermanitos menores. }\end{array}$ & $\begin{array}{l}\text { Pucllacoc huarmi huanra. En lo mismo que } \\
\text { los varoncitos y, además, en las labores de la } \\
\text { cocina. Tambien como pajes de las señoras } \\
\text { nobles. }\end{array}$ \\
\hline De 9 a 12 años & $\begin{array}{l}\text { Tocllayoc huarnacuna. Caza de aves. Obtención } \\
\text { de plumas. Hilanderia. Pastores. Leñadores. } \\
\text { Muchachos de mano o mandaderos de las } \\
\text { autoridades. }\end{array}$ & $\begin{array}{l}\text { Pagua pallac. Recojo de flores tintoreras. } \\
\text { Recolecta de yerbas para la dieta familiar y de } \\
\text { los señores. Podían ser llevados a la capacucha: } \\
\text { sacrificios humanos. }\end{array}$ \\
\hline De 12 a 18 años & $\begin{array}{l}\text { Mactacuna. Caza de aves. Disección de su carne. } \\
\text { Obtención de plumas. Participación en el ayni } \\
\text { interfamiliar y mingas del ayllu. Servicio al curaca. }\end{array}$ & $\begin{array}{l}\text { Rotuscatasca. Hilado. Pastoreo. Tareas } \\
\text { agrícolas en las tierras de los señores. }\end{array}$ \\
\hline De 18 a 25 años & $\begin{array}{l}\text { Sayapayac. Chasquis. Pastores. Guerreros. Mita- } \\
\text { yos. }\end{array}$ & $\begin{array}{l}\text { Allin suma sipascuna. Acllas para ser regaladas } \\
\text { a los nobles y otros privilegiados. }\end{array}$ \\
\hline De 25 a 50 años & $\begin{array}{l}\text { Aucamayoc. Mita agrícola. Artesanos. Mineros. } \\
\text { Ejército. Mitmas. }\end{array}$ & $\begin{array}{l}\text { Aucamayo huarmin. Tejedoras de cumbi } \\
\text { (tapicería) para el Estado. }\end{array}$ \\
\hline De 50 a 80 años & $\begin{array}{l}\text { Puricmachus. Leñadores. Servicio de limpieza en } \\
\text { las casas de los nobles. Camareros. Despenseros. } \\
\text { Porteros. Quipucamayos. Lacayos de la aristocracia. }\end{array}$ & $\begin{array}{l}\text { Payac-cuna. Tejedoras de abasca para el } \\
\text { Estado. Porteras. Despenseras. Camareras. } \\
\text { Cocineras. Mayordomas. Criadas de acllas. }\end{array}$ \\
\hline De 80 a más años & $\begin{array}{l}\text { Roctomachu. Por lo general descansaban, pero } \\
\text { quien podía trenzaba sogas, cuidaba conejos y patos. } \\
\text { Se les preferia como porteros de los acllahuasis y } \\
\text { casas de coyas y señoras mamacuracas. Otros } \\
\text { trabajaban en sus chacras. Eran los narradores de } \\
\text { mitos, leyendas y cuentos. La comunidad les pedia } \\
\text { su consejo. }\end{array}$ & $\begin{array}{l}\text { Puñocpaya. Por lo usual no hacían nada. Pero } \\
\text { a quienes podían dedicabanlas como porteras, } \\
\text { acompañantes, tejedoras de costales, criadoras } \\
\text { de conejos y patos, cuidadoras de niños. } \\
\text { Despemnseras y porteras de las señoras de la } \\
\text { aristocracia regional y estatal. }\end{array}$ \\
\hline De cualquier edad & \multicolumn{2}{|c|}{$\begin{array}{l}\text { Aquí estaban comprendidos los enfermos crónicos, lisiados, cojos, mancos, tullidos, mudos, ciegos, } \\
\text { enanos baldados, idiotas u opas y locos, tanto de sexo masculino como del femenino. Sin embargo, no } \\
\text { se les dispensaba a quienes podían desempeñarse en actividades apropiadas a su estado. Los enanos } \\
\text { por ejemplo, en la bufonería y chocarrería; los mancos como lazarillos; los tullidos como quipucamayos y } \\
\text { tejedores. Los enanos con dotes histriónicos eran reclutados por orden del sapainca para que divirtieran } \\
\text { a sus esposas y otras señoras de la corte. A los enanitos jorobados se les preferia como pajes de señoras } \\
\text { y señores por creer que traían suerte. Precisamente el dios Equco, que velaba por la buena fortuna de } \\
\text { la gente, tenía figura de jorobado. } \\
\text { Lo mismo sucedia con las mujeres pertenecientes a este grupo. A las que podían se las encargaba } \\
\text { labores textiles, de bordaduría, confección de chumbis y huinchas, preparación de potajes en las cocinas } \\
\text { de algunas familias nobles, a las que se las donaba. }\end{array}$} \\
\hline
\end{tabular}

Fuente: Waldemar Espinoza Soriano. Los Incas. Economía, sociedad y estado en la era del Tahuantinsuyo. 1987. Pag. 221 - 222 (6)

\section{EL PROCESO DE FORMACION DEL RUNA EN LOS ANDES}

La palabra runa ("ser humano") es usada indistintamente para varones y mujeres desde el momento de la concepción.
La gestación es percibida como el proceso de absorción "devoradora" de la sangre de la madre por el feto, quien es percibido como poseído por una entidad ancestral, peligrosa y agresiva, con quien la madre debe "luchar" hasta el momento del parto. Desde el nacimiento, la madre retrasa la hora de dar pecho al niño para subordinar su actitud "devoradora" y disciplinarlo; 
incluso, la madre puede mostrarse severa con respecto al llanto del niño, y regañarlo con la voz. Durante todo el periodo de lactancia hasta el destete, el niño se considera vulnerable a todo tipo de espíritus malhechores capaces de llamarlo al otro mundo a través de las enfermedades o la muerte, por lo que la madre está atenta a ver si el niño levanta y sostiene la cabeza, puede sentarse, caminar y alimentarse. Las madres saben que a partir del sexto mes los niños enflaquecen, por lo que una baja de peso o talla por separado no es considerado como un signo de alarma. Lo que ellas observan es si el niño es bien proporcionado. En esta etapa, el niño depende de su madre, quien lo amamanta de manera regular y "a pedido" (8).

Cada runa, en cada etapa del ciclo vital en que se encuentre, asume roles que implican obligaciones y responsabilidades. Se pierde la calidad de runa, sin dejar de ser humano, si no se ejerce los cargos tradicionales, si no se casan, si no tienen hijos, y si no cumplen sus obligaciones y responsabilidades que les compete en cada etapa.

En 1970, en base a su experiencia en Chuschi Ayacucho, Isbell (9) presenta su teoría de los tropos de género, conceptualizando que el paso del nacimiento a la muerte es un proceso "de infantes inmaduros a antepasados petrificados", similar al que sigue la crianza de la papa. Isbell, divide este proceso en 5 etapas:

1 La infancia inmadura. La vida empieza en un estado húmedo, "inmaduro", femenino. Al recién nacido se le llama llullu wawa, o wawa mojada, verde, inmadura. Los niños son fajados para que sus miembros crezcan rectos y fuertes. Recibe los cuidados maternos, pero con aparente poca atención afectiva como probable mecanismo de protección de los padres ante el trauma de la mortalidad infantil. Cuando pueden sentarse y caminar, abandonan el término Ilullu: Sus padres les llaman warmi churi (hija mujer) o qari churi (hijo varón), según la diferencia de género, pero sus madres les llaman indistintamente wawa.

2 Caminando hacia la niñez y el mundo del trabajo. Al empezar a caminar, la ropa y peinados es específica para cada sexo (pantalón los varones y ropa de mujer para niñas). Hasta los 5 o 10 años son llamados warma por ambos padres, que puede ser traducido como niño (a). Empiezan a ser socializados en el trabajo específico para cada género, primero en la unidad doméstica y luego en el cuidado de animales en los pastizales, alrededor de los 7 u 8 años.

3 La adolescencia. Se utiliza los términos Sipas para chicas que aún no han iniciado su actividad sexual. Una vez que inician su actividad sexual se les llama Pasña. En el caso de los varones, se usa el termino maqta, sean sexualmente activos o no.

4 La madurez. El estado adulto se define culturalmente con el matrimonio. Las mujeres experimentan la presión social para casarse y reproducir la sociedad. La palabra warmi, implica, hija, mujer y esposa. Una mujer casada que no tiene hijos se le considera esposa del wamani o deidad de los cerros. Los varones solteros son ridiculizados como niños perpetuos. A los hombres como maridos se les llama qosa, y a los hombres en general qari.

5 La vejez. Tiene un carácter masculino para ambos sexos. El término para una mujer postmenopáusica es paya. Machu significa hombre viejo y animal macho. Los ancianos pierden su humedad y se convierten en chuñu, como las papas disecadas, pierden humedad y se petrifican. La muerte trae la desecación final. Dicen que los muertos humanos son chuñu, aptos para el almacenamiento y uso futuro, en la idea que los muertos petrificados contribuyen a la esencia de los recién nacidos.

En 1999, en Ninamarka-Cusco ${ }^{(10)}$, se identificaron las siguientes etapas de desarrollo humano:

\section{A. WAWA, PRIMERA ETAPA DEL CRECIMIENTO Y DESARROLLO DEL NIÑO ANDINO}

El termino wawa es usado por las mujeres para designar un niño o bebé ${ }^{(11)}$. Este término también se usaba para designar a un hermano(a) materna ${ }^{(12)}$. Según Zuidema, desde el tiempo de los incas, una madre utiliza la palabra wawa para sus hijos, sean hombres o mujeres ${ }^{(13)}$. Para Rengifo ${ }^{(14)}$, la palabra no se refiere a un individuo. Expresa que es fruto de la vinculación exitosa de sus padres biológicos, así como la vinculación de la madre con sus hijos (sean hombres o mujeres), y con las deidades protectoras de la comunidad.

Sean varones o mujeres, desde el nacimiento hasta que sus huesos, músculos y articulaciones adquieran cierta consistencia, se les denomina Ilullu wawa ("bebé tierno que aún no termina de madurar"). Este periodo puede ser variable, se extiende aproximadamente hasta los tres meses de edad. No se aprecia que las madres establezcan diferencias entre el llullu wawa varón y la Ilullu wawa mujer. La madre es la única que cuida al bebé en esta etapa de la vida, porque consideran que su cuerpo es muy frágil y que tienen el riesgo de aysarukun (deshacerse, romperse en pedazos, fracturarse), por lo que son fajados. La madre por ningún motivo encarga el cuidado del qhulla wawa a alguna de sus hijas.

Luego, el bebe deja de ser llamado qhulla wawa para recibir la denominación de ñuñuq wawa ("bebé que lacta"), 
que vive exclusivamente de leche materna. No solo lacta por el estímulo de la madre, el infante toma la iniciativa de lactar, lo exige con sus llantos y busca la mama de la madre para lactar levantándole la blusa. Desde aproximadamente los seis meses de edad empieza a probar los primeros alimentos en su sabor, textura o consistencia. Su cuerpo adquiere estabilidad y es posible que una de las hermanas mayores lo cuide. Esta etapa, en promedio, se extiende desde los tres meses hasta por lo menos el año y medio o dos años.

Finalmente, en la fase del p'iti wawa "(niño destetado"), - puchu wawa, se ha independizado de la leche materna, ingiere alimentos sólidos y tiene la habilidad de caminar solo. Empieza a valerse por sí mismo en su cuidado personal, vestido, alimentación y sueño. Esta etapa en promedio, se extiende hasta los cinco años de edad, y utilizan la phalika (faldín que se amarra a la cintura del varón o la mujer) porque les facilita hacer sus necesidades fisiológicas (orina y deposiciones).

En esta etapa no se le atribuye ninguna premeditación en sus actos, por lo que el castigo es considerado innecesario. Alrededor de los 2 o 3 años realizan el primer corte de pelo de los niños. Representa la continuidad de un rito de pasaje que se realiza desde el tiempo de los incas. Se socializa, desarrolla habilidades lingüísticas y se individualiza como miembro de la familia.

\section{B. EL SER HIRQUI}

Un hirqui tiene el cuerpo más desarrollado y presenta yuyayniyuq (raciocinio), por lo que es sujeto de obligaciones y derechos. Puede realizar tareas que le asignan sus padres, quienes le enseñan que debe mejorar su vida con sus estudios y trabajo, haciendo bien las cosas, siendo colaborador y responsable. Consideran que aún posee inocencia y que no es capaz de medir las consecuencias de sus actos. No necesita tanto cuidado como un wawa, pero necesita ser bien alimentado para que su cuerpo desarrolle. Es educado en roles y labores propias de un varón o mujer, según corresponda, aprendiendo a sacar adelante una granja y un hogar. Poco a poco se va independizando del cuidado paterno. El juego con pares y el desarrollo en equipo de las tareas asignadas se hace importante. Se considera que desde los 4 años los niños empiezan a trabajar. Aunque la edad más mencionada es a los 6 o 7 años ${ }^{(15)}$. Esta etapa se prolonga hasta los 13 a 14 años.

\section{PASÑA Y MAQTA}

Con la menstruación, la mujer adquiere el estatus de pasña, que es equivalente de adolescente. Realiza sus tareas sin necesidad que le estén recordando u ordenando. Por sí sola se organiza, incluso con su propio fiambre y tejidos. Enfatiza el desarrollo de habilidades textiles y domésticas. Esta etapa termina aproximadamente a los 16 años.

El Makta se inicia cuando es visible la aparición de vellos en el cuerpo y el cambio de voz. A partir de ahí, maktas y pasñas se separan para formar grupos con pares del mismo sexo. Empieza el proceso de enamoramiento, como paso previo a la elección de esposo o esposa. En los varones, se valora su capacidad para obtener el sustento familiar o "ganarse la vida".

\section{SIPAS Y WAYNA}

El makta pasa a ser wayna cuando su cuerpo adquiere fortaleza muscular visible, y demuestra que puede resistir todo un día de trabajo en la chacra o en el traslado de objetos pesados. Solo quienes reúnen estas características, se estima están en condiciones de tener pareja. La sipas o el wayna, ya poseen yuyayniyuq y su conducta y decisiones depende de cómo lo usen. Saben lo que es bueno y malo, lo que debe y no debe hacer un/a joven. Son responsables y no necesitan que sus padres les estén ordenando. De no actuar correctamente, no serían potenciales elegibles para esposos/as. Su cuerpo está listo para procrear hijos. Están listos para formar pareja y hacerse responsables de sus vidas y los hijos que tendrán. El ideal es que reflexione sobre lo que es bueno para él/ella y la comunidad, como lo haría un allin runa.

Las sipas, en promedio, se mantienen como tal hasta los 20 años, aproximadamente. Cuando pasan esta edad y no han conseguido establecer una vida conyugal les dicen "paya soltera" (soltera vieja) y consideran que se quedará así a menos que la "recoja" un viudo. El wayna, si no consiguen tener pareja hasta los 22 años, aproximadamente, es denominado "machu soltero" (soltero viejo). Tiene la opción de conseguir pareja en otra comunidad, siempre y cuando una sipas le acepte. Un soltero es considerado un ser humano incompleto, al igual que las parejas sin hijos (as). Un hombre o una mujer sin hijos nunca llegan a gozar del pleno estatus dentro del grupo.

\section{E. WARMI Y QHARI}

La warmi tiene que cumplir con sus nuevas obligaciones y responsabilidades de esposa, al igual que el qhari. En los andes, la pareja, y no el individuo, constituye la unidad social basica:

La pareja andina es el núcleo ideológico del sistema de parentesco de la tradición cultural que conjugan los quechuas, los aimaras y los yungas, a diferencia de Europa occidental donde parece ser, más bien, la filiación. Esta relación por afinidad es lo fundante en 
Tabla 2. Comparación de las etapas del desarrollo: Freud, Erikson, andes

\begin{tabular}{lccc}
\hline Edad aproximada & Freud & Erikson & Andes \\
\hline Del nacimiento a 1 año & Etapa oral & Confianza contra desconfianza & Wawa \\
1 a 2 años & Etapa anal & Autonomía contra vergüenza y duda & Wawa \\
\hline a 5 años & Etapa fálica & Iniciativa contra culpa & Wawa \\
6 a 11 años & Etapa de latencia & Industria contra inferioridad & Hirqui \\
12 años a la juventud & Etapa genital & Identidad contra confusión de roles & Pasña/Makta \\
Juventud & Etapa genital & Intimidad contra aislamiento & Sipas/Wayna \\
Madurez & Etapa genital & Generatividad contra estancamiento & Warmi/Qari \\
Vejez & Etapa genital & Industria contra desesperación & Paya/Yuyak \\
\hline
\end{tabular}

Fuente: Rice, F. Philip, Desarrollo Humano: Estudio del Ciclo Vital 1997: 35. (25). Adaptación propia.

el mundo andino, aquello que da plenitud social, y no tanto, la prolongación de la vida biológica a través de las generaciones. La persona, social y moralmente plena, tiene pareja, y gracias a ella, es padre, madre, pasa cargos religiosos y políticos ${ }^{(16)}$.

La adultez es el vínculo entre el ciclo vital individual y el ciclo de las generaciones ${ }^{(17)}$.

\section{F. PAYA Y YUYAQ}

La etapa de los yuyak ("El que recuerda") se da cuando tanto warmi como qhari empiezan a envejecer y muchos de sus hijos ya forman otras parejas fuera de la casa paterna. Empiezan a ayudar en la crianza de los nietos. Conversan de sus experiencias y acontecimientos comunales pasados. Se prefiere usar la palabra yuyak en reemplazo de machu para los varones y paya para las mujeres, porque consideran denigrantes estos términos.

\section{DISCUSIÓN}

Como puede apreciarse, el ciclo vital no es un fenómeno inmutable, el número de etapas y su significado está pautado histórica y culturalmente. Por ello, se ha considerado que culturas diferentes darían lugar a personas diferentes ${ }^{(18)}$, y que la psicología occidental es irrelevante para Asía, donde la unidad social la constituye la familia y no el individuo ${ }^{(19)}$.

Del análisis comparativo de Tabla 2, se tiene, que el psicoanálisis, con su orientación clínica, se centra en el desarrollo de la personalidad individual ${ }^{(20)}$, considerando al individuo como un haz de instintos que buscan ser satisfechos ${ }^{(21)}$. Erikson concibe el desarrollo humano como producto de la interacción de múltiples factores internos y externos que confluyen en la interacción familiar ${ }^{(22)}$. Por su experiencia con los sioux, concluyó que la crianza es la forma en que el ethos del grupo es transmitido al infante ${ }^{(23)}$, lo que explicaría la resistencia de los sioux a asimilar esquemas occidentales: sus sistemas de enseñanza tradicionales han cambiado muy poco, por lo que su personalidad y cultura ha cambiado solo en parte ${ }^{(24)}$. En este debate, el aporte de la cultura andina, se centra en: 1) Cada etapa del desarrollo humano está asociada a procesos productivos y reproductivos de la vida en relación, así como a un adecuado equilibrio entre derechos y deberes en la vida en sociedad, 2) La pareja es la unidad social básica, y no el individuo.

Fuentes de financiamiento: autofinanciada.

Conflictos de interés: no hay conflictos de interés en la publicación de este artículo.

\section{REFERENCIAS BIBLIOGRÁFICAS}

1. OPS/OMS. Derechos, salud intercultural y políticas públicas. Baca, María Edith. Recuperado de: http://www. cultura.gob.pe/sites/default/files/ paginternas/tablaarchivos/2013/09/ derechossaludinterculturalypolticaspublicasmariaedithbacacabrejosperu.pdf

2. Bonnet D. Argumentos para un enfoque global de la pediatría. Salud, crecimiento y desarrollo del niño. En: Miradas cruzadas en el niño. Un enfoque interdisciplinario para la salud, el crecimiento y el desarrollo del niño en Bolivia y Perú. Editores: Charles-Edouard de Suremain, Pierre Lefevre, Emma Rubin de Celis, Edgar Sejas. Instituto Frances de Estudios Andinos. Institut de Recherche pour le Developpment. Plural editores. Impreso en Bolivia. 2003. Pág.: 10

3. Jaes C. Contribuciones de la sociología de la familia y de la terapia familiar al "esquema del desarrollo familiar": análisis comparativo y reflexiones sobre las tendencias futuras. En: Transiciones de la familia. Continuidad y cambio en el ciclo de vida. 1991. Amorrortu editores. Buenos Aires. Argentina.1991. Pág.66.

4. Boggio C. Estudio del Ciclo Vital en Pamplona Alta. Centro de Estudios y Promoción del Desarrollo (Desco). Lima, Perú.1968. Pág.5. 
5. Gonzales-Carre E, Galdo-Gutierrez V. Introducción al proceso de socialización andina. Universidad Nacional de San Cristóbal de Huamanga. Serie Tricentenario 1677 1977. Ayacucho, Perú.1976. Pág.4.

6. Espinoza-Soriano W. Los Incas. Economía, Sociedad y Estado en la Era del Tahuantinsuyo. Amaru Editores. Primera Edición. Impreso en Lima, Perú. 1987. Pág. 221-222.

7. Salazar-Soler C, Lestage F. Grupos de edad en la visita de Huánuco. En: Arnold, Denise Y. Gente de carne y hueso. Las tramas de parentesco en los andes. Tomo II. Biblioteca de Estudios Andinos. ILCA/CIASE Research Series $N^{\circ} 28$. 1998. La Paz, Bolivia.1998. Pág. 370.

8. Bonnet D. Argumentos para un enfoque global de la pediatría. Salud, crecimiento y desarrollo del niño. En: Miradas cruzadas en el niño. Un enfoque interdisciplinario para la salud, el crecimiento y el desarrollo del niño en Bolivia y Perú. Editores: Charles-Edouard de Suremain, Pierre Lefevre, Emma Rubin de Celis, Edgar Sejas. Instituto Frances de Estudios Andinos. Institut de Recherche pour le Developpment. Plural editores. Bolivia. 2003. Pág.13-14.

9. Isbell BJ. De inmaduro a duro: Lo simbólico femenino y los esquemas andinos de género. En: Arnold, Denise Y. Más allá del silencio. Las fronteras de género en los andes. Tomo I. Biblioteca de Estudios Andinos. ILCA/CIASE Research Series N ${ }^{\circ} 28$. 1997. La Paz, Bolivia.1997. Pág. 293-298.

10. Santa María Juárez LA. Sistema Cultural de Crianza en Ninamarca, Cusco. Tesis para optar el Grado de Doctor en Antropologia. UNMSM. 2017. Pág. 570 - 616 .

11. Isbell BJ. Estructura del parentesco y del matrimonio. En: Parentesco y matrimonio en los Andes. E.Mayer, R. Bolton Editores. Pontificia Universidad Católica del Perú. Fondo Editorial. 1980. Lima, Perú. 1980. Pág. 207.

12. Ellefsen B. Matrimonio y sexo en el incario. Enciclopedia boliviana. Editorial Los Amigos del Libro. Cochabamba, La Paz - Bolivia. 1989. Pág. 66.

13. Zuidema RT. Parentesco Inca. El sistema de parentesco incaico: una nueva visión teórica. En: Parentesco y matrimonio en los Andes. E.Mayer, R. Bolton Editores. Pontificia Universidad Católica del Perú. Fondo Editorial. 1980. Lima, Perú. 1980. Pág. 64.

14. Rengifo G. Concepción andina sobre la niñez. Ser wawa en los andes: Aproximaciones. En:Qinasay, Revista de Educación Intercultural Bilingüe. $\mathrm{N}^{\circ}$ 4. Junio 2006. Pag. 175 - 180. Universidad Mayor de San Simón Cochabamba, Bolivia. 2006. Pág. 176.

15. Bolton C, Bolton R. Técnicas de socialización entre los qolla. En: La vida familiar en comunidades andinas. Estudios antropológicos en la sierra sur del Perú. Antropología y Etnología 10. Primera Edición. Editorial Horizonte. Lima, Perú. 2010. Pág. 244.

16. Ortiz A. Pobres y Ricos. La pareja en el mundo andino. En: El amor y sus especies. Liuba Kogan, Augusto Castro, Alejandro Ortiz, Carlos Cáceres, Ana Lydia Vega. Facultad de Ciencias Sociales. Pontifica Universidad Católica del Perú. Colección temas en sociología $\mathrm{N}^{\circ}$ 4. Lima, Perú. 1995. Pág.33

17. Erikson E. El ciclo vital completado. Edición revisada y ampliada. Paidos. Primera Edición. Tercera impresión. Impreso en España. 2011. Pág. 20.

18. Garrido MJ. Etnopediatria en contextos virtuales. Un nuevo paradigma social y antropológico basado en la crianza respetuosa y su articulación en internet. Tesis Doctoral. Universidad de Extremadura. Departamento de Psicologia y Antropologia. Recuperado de: http://dehesa.unex. es/handle/10662/144. 2012. Pág. 36.

19. Majluf A, Ojeda G. Relación entre tradicionalismo y prácticas de crianza. En: Revista Psicológica Herediana. Universidad Peruana Cayetano Heredia. Año $\mathrm{N}^{\circ} 1, \mathrm{~N}^{\circ} 1$. Mayo 2006. Lima, Perú. 2006. Pág.31.

20. Elkin F. El niño y la sociedad. Paidós educador. Decima reimpresión. Buenos Aires, Argentina. 1989. Pág. 53-54.

21. Rochabrun G. Socialidad e individualidad. Materiales para una sociología. Segunda edición. Fondo editorial. Pontificia Universidad Católica de Perú. Lima, Perú.1993. Pág.102, 112.

22. Caballero N. Patrones de crianza infantil. Pontificia Universidad Católica del Perú. Lima, Perú. S/D. Pág. 11.

23. Erikson E. El ciclo vital completado. Edición revisada y ampliada. Paidos. Primera Edición. Tercera impresión. Impreso en España. 2011. Pág. 30.

24. Tentori J. Antropología cultural. Editorial Herder. Barcelona, España. 1980. Pág.14.

25. Rice FP, Desarrollo Humano: Estudio del Ciclo Vital 1997. Pág. 35.

Correspondencia: Luis Santa María.

Dirección: Jr. Capac Yupanqui 1400, Lima 11

Correoelectrónico:luchins@hotmail.com

\section{Nuestros artículos se encuentran indizados en:}

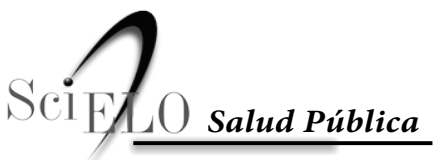

www.scielo.org 